\title{
Evolution of Interfacial Curvatures of a Bicontinuous Structure Generated via Nonconserved Dynamics
}

\author{
C.-L. Park ${ }^{\mathrm{a}}$, P. W. Voorhees ${ }^{\mathrm{b}}$, K. Thornton ${ }^{\mathrm{a}, *}$ \\ ${ }^{a}$ Department of Materials Science and Engineering, University of Michigan, 2300 Hayward, Ann Arbor, \\ MI 48109, USA \\ ${ }^{b}$ Department of Materials Science and Engineering, Northwestern University, 2220 Campus Drive, \\ Evanston, IL 60201, USA
}

\begin{abstract}
While coarsening of spherical particles has been well documented, our understanding of coarsening of complex microstructures is still limited. The first step in developing a theory of coarsening of microstructures with complex morphologies is to study coarsening of microstructures that evolve self-similarly. In this paper, we examine the morphological evolution of a self-similar two-phase bicontinuous structure generated via nonconserved dynamics (i.e., motion by mean curvature) to elucidate the complex dynamics of coarsening. We find that the evolution proceeds with some interfaces evolving toward topological singularities (pinching) while the majority of interfaces flatten. These two processes were also illustrated through the evolution equation for the mean curvature, which has a term that depends solely on the local curvatures, as well as a term that is proportional to the surface Laplacian of the mean curvature. The first term causes an increase in the magnitude of the mean curvature, while the second term causes smoothing of the mean curvature in a manner similar to diffusion of chemical species on a surface. The second term causes a large dispersion in the values of the time derivative of mean curvature at various locations in the structure, characterized neither by the mean curvature nor the Gaussian curvature. Keywords: coarsening, bicontinuous structure, mean curvature, Gaussian curvature, phase-field method
\end{abstract}

${ }^{*}$ Corresponding author: Email address: kthorn@umich.edu, Tel: +17346151498 


\section{Introduction}

Coarsening is a ubiquitous natural phenomenon that occurs in a wide range of materials, such as metallic alloys $[1,2,3,4,5,6,7,8]$, polymers $[9,10,11,12,13,14,15]$, and semiconductors $[16,17,18,19,20,21]$. During coarsening, the total interfacial area of a microstructure decreases to reduce excess free energy associated with the existence of phase boundaries. Therefore, coarsening can play a key role in determining the morphology and the topology of the microstructure of many materials. Coarsening is preceded by phase separation, where a single-phase system transforms into a two-phase system. Regardless of whether the phase transformation is continuous, as in spinodal decomposition, or discontinuous, as in nucleation [22], once the concentration of the newly formed phases are close to their equilibrium values, the microstructure begins to coarsen.

Study of coarsening is important since the microstructure of a material often has a strong influence on its properties. As an example, during casting of metallic alloys, solidliquid dendritic mixtures frequently experience temperatures above the eutectic temperature $[2,23,24,25,26,27]$. If the removal of heat is sufficiently slow, the dendrites begin to coarsen. The resulting evolution of the shape and size distributions of the dendrites during coarsening alters the properties of the cast alloy. In the case of an Al-Si cast alloy, for example, as the secondary dendritic arm spacings increase, the corrosion resistance improves while the tensile strength decreases $[28,29]$. This evidence suggests that the understanding of the dynamics of coarsening is essential for engineering materials with desired properties.

Coarsening has been well studied in particle systems [30]. The classical theory developed by Lifshitz and Slyozov [31], and Wagner [32] (LSW) predicts that the particle size distribution (PSD) of the particle system undergoing coarsening becomes time-independent when scaled by the time-dependent average particle size, which grows as $t^{1 / 3}$. Most microstructures, however, are non-particulate and have spatially varying interfacial curvatures within individual domains. The complex interfacial morphologies make it difficult to elucidate the dynamics of coarsening in microstructures with intricate morphologies.

The development of theory of coarsening in complex microstructures can be facilitated 
if the microstructure evolves self-similarly, i.e., the interfacial morphologies become timeindependent when scaled by a time-dependent characteristic length scale. Self-similar structures are ideally suited for developing theoretical understanding since they possess a singlemodal distribution of features and the governing equation becomes time independent when scaled with the characteristic length scale.

Two types of complex microstructures that evolve self-similarly are the bicontinuous structures that result from phase decomposition such as spinodal decomposition [33, 34, 35, $36,37,38,39,40,41]$ and phase ordering [42, 43, 44, 45, 46, 39, 40]. In a bicontinuous structure, two phases interpenetrate one another and are each connected to itself throughout. Physically, these bicontinuous structures can form during phase-ordering in magnetic materials $[42,43,44,46]$, spinodal decomposition in polymer mixtures $[33,34,36]$, and in microemulsions [37, 38]. Kwon et al. confirmed that the bicontinuous structures simulated via conserved (spinodal decomposition) and nonconserved (phase-ordering) dynamics evolve self-similarly during coarsening [39, 40]. Nonetheless, our understanding of the dynamics of coarsening in these bicontinuous structures is still limited, primarily because previous analyses have been based on the morphological properties of interfacial characteristics (hereafter, simply referred to as interfacial properties) that are static measurements. We believe that the dynamics of coarsening should be investigated with both static and dynamic measurements of interfacial properties that can better quantify the morphology of a microstructure and its evolution.

Despite the relative simplicity of the problem as compared to structures that involve multiple feature sizes (such as dendritic structures), the theoretical study of the dynamics of coarsening is still challenging because bicontinuous structures inherently possess multiple length scales, as the interfaces have two independent curvatures. Thus, we must develop a framework for theoretical development. As was done in LSW theory, we characterize the microstructure statistically, but in curvature space, using the Interfacial Shape Distribution (ISD) $[39,40]$. We also propose to consider the evolution as a consequence of (1) the interfacial velocity induced by diffusion and (2) the resulting evolution of the interfacial curvatures. In this paper, we focus on (2) the evolution of interfacial curvature given 
the interfacial velocity. Using the phase-field method, a bicontinuous two-phase structure resulting from nonconserved (Allen-Cahn) dynamics is obtained (which will hereafter be referred to as the Allen-Cahn structure). In this dynamics, the interfacial velocity is directly proportional to the mean curvature (i.e., the structure evolves by "motion by mean curvature"), and therefore it provides an ideal test bed with which to investigate the curvature velocity. We will compute and analyze the interfacial properties and their rate of change (time derivatives). We present local and statistical analyses on the evolution of interfacial curvatures of the Allen-Cahn structure to provide insights into the dynamics of coarsening that are possible only with the incorporation of both the static and dynamic measurements of interfacial properties.

\section{Computational Methods}

This section briefly describes the phase-field method used to simulate the bicontinuous Allen-Cahn structure and the level-set smoothing method employed to smooth the microstructural data obtained from the phase-field method for accurate calculations of interfacial properties.

\subsection{Phase-Field Method}

The Allen-Cahn structure is simulated using the phase-field method. The phase-field method belongs to the diffuse interface approach, which eliminates the need for explicitly tracking the locations of interfaces with markers. This feature makes the phase-field method an attractive computational tool for studying microstructural evolution with complex interfacial motions. While other simulation techniques such as the level-set method $[47,48]$ can be applied to simulate mean curvature flow, we employ the phase-field approach based on Allen-Cahn dynamics because it maintains equal volume fractions in the structure we examine without any special treatment and because we have highly parallel computational software infrastructure already developed.

In the phase-field method, different phases are represented by the bulk values of the order parameter, $\phi$, and the phase boundaries are described by the smooth transition of the value 
of $\phi$ between these bulk values. The governing equation for nonconserved (Allen-Cahn) dynamics used to generate the Allen-Cahn structure is [49]

$$
\frac{\partial \phi}{\partial t}=-L\left(\frac{\partial f}{\partial \phi}-\epsilon^{2} \nabla^{2} \phi\right)
$$

The bulk free energy, $f(\phi)$, is approximated as a double-well potential,

$$
f(\phi)=\frac{W}{4} \phi^{2}(1-\phi)^{2}
$$

an expression which is often used for the conserved (Cahn-Hilliard) equation and has minima at $\phi=0$ and 1 (corresponding to two bulk phases) [50,51]. Note that, for many nonconserved systems, the expression is often taken such that the two bulk phases have $\phi$ values of -1 and 1 instead. Variables $L, \epsilon, W$ are the mobility coefficient, the gradient energy coefficient, and the height of the double-well potential, respectively. We use the dimensionless form of the Allen-Cahn equation, and therefore all measurements of length and time are dimensionless. A grid spacing of $\Delta x=\Delta y=\Delta z=1.0$ and a time step of $\Delta t=0.1$ with $L_{\phi}=1.0$ are employed for simplicity. The remaining parameters are $\epsilon^{2}=0.4$ and $W=0.8$, which leads to an interfacial thickness of $\delta=4$ and interfacial energy of $\gamma=0.2$. To generate sufficient statistics, a large computational domain size of $2048 \times 2048 \times 2048$ in a Cartesian-grid system is simulated. Starting from an initial condition consisting of random numbers that vary between $\phi=0.5 \pm 0.1$ that ensures equal volume fractions of the two phases, the simulation was run until the bicontinuous structure undergoes sufficient amount of coarsening to achieve self-similarity, as determined by absence of evolution of the scaled ISD. Even though the dynamics is noncoserved, the system maintains a 50:50 volume fraction due to its symmetry. Fig. 1 shows the morphology of a portion (1/512 of the entire volume) of the Allen-Cahn structure at nondimensional simulation time $t_{0}=600$ (note it is not possible to display the entire volume with adequate resolution); however, the analyses utilize the entire simulated volume. The data from this simulation time $\left(t_{0}=600\right)$ is used for all analyses presented in this paper. 


\subsection{Level-Set Smoothing Method}

To achieve sharp-interface asymptotics while controlling computational costs, the width of the diffuse interface of the Allen-Cahn structure is described by four Cartesian grid points. The interfacial resolution of the Allen-Cahn structure is inadequate to accurately evaluate the high-order derivatives required for the calculation of interfacial properties. Therefore, we apply the level-set smoothing method [52] on the order parameter obtained from the phase-field simulation that describes the Allen-Cahn structure. The level-set smoothing method is a set of sequential data-processing schemes that consists of first generating the signed distance function for the given microstructure using the level-set method, followed by smoothing via diffusion. Full details of the application of the level-set smoothing method to the microstructural data describing the Allen-Cahn structure can be found in Ref. [52]. After applying the level-set smoothing, the Allen-Cahn structure is described by the smoothed level-set function.

\subsection{Calculation of Interfacial Properties}

We characterize the interfacial morphology of the Allen-Cahn structure with the mean and the Gaussian curvatures, $H$ and $K$, respectively, or alternatively with the minor and major principal curvatures, $\kappa_{1}$ and $\kappa_{2}$, respectively. The mean and Gaussian curvatures are calculated using the level-set approach $[47,52]$. The two principal curvatures are then calculated from the expressions:

$$
\kappa_{1}=H-\sqrt{H^{2}-K}, \quad \kappa_{2}=H+\sqrt{H^{2}-K} .
$$

The normal displacement of interfaces is characterized with the interfacial normal velocity, $v$, which is also calculated using the level-set approach $[47,52]$. On the other hand, the evolution of interfacial morphology is characterized with the Lagrangian time derivatives of

the mean and Gaussian curvatures, $\dot{H}$ and $\dot{K}$, respectively, or alternatively with the time derivatives of the principal curvatures, $\dot{\kappa}_{1}$ and $\dot{\kappa}_{2}$. These dynamic interfacial properties are calculated from the smoothed level-set function describing the microstructure at different 
simulation times. The time derivatives of curvatures are calculated using the surface-meshbased advective method in the moving frame of the interface [52]. The time derivative of the mean curvature, $\dot{H}$, for example, is expressed as:

$$
\dot{H}\left(\boldsymbol{x}\left(t_{1}\right), t_{1}\right)=\frac{H\left(\boldsymbol{x}\left(t_{2}\right), t_{2}\right)-H\left(\boldsymbol{x}\left(t_{1}\right), t_{1}\right)}{\delta t},
$$

where

$$
\boldsymbol{x}\left(t_{2}\right)=\boldsymbol{x}\left(t_{1}\right)+\boldsymbol{v} \delta t
$$

Here, $\boldsymbol{v}$ is the normal velocity vector defined as the product of the interfacial velocity, $v$, and the unit normal vector, $\boldsymbol{n}, \boldsymbol{x}\left(t_{1}\right)$ is the interfacial coordinate at reference simulation time $t_{1}, \boldsymbol{x}\left(t_{2}\right)$ is the predicted interfacial coordinate of the point $\boldsymbol{x}\left(t_{1}\right)$ at time $t_{2}$ (see Eq. (5)), and $\delta t$ refers to a time interval used in the calculation of time-derivative quantities, which depends on the surface curvature. The full details of the method for calculating the timederivative quantities are described in Ref. [52]. We note that the computed time-derivative quantities will incur substantial errors near singularities (like pinching); however, they occur quickly and involve very small regions, and therefore are not expected to influence the overall results. All interfacial properties presented in this paper are scaled by the characteristic length scale, $S_{v}^{-1}$, and the corresponding time scale, $S_{v}^{-1} /\left(d S_{v}^{-1} / d t\right)$, where $S_{v}$ is the surface area per unit volume calculated for the entire simulation volume. For example, $H=\tilde{H} S_{v}^{-1}$ and $v=\tilde{v} /\left(d S_{v}^{-1} / d t\right)$, where $\tilde{H}$ and $\tilde{v}$ are the numerically calculated mean curvature and interfacial velocity, respectively.

\section{Procedures for Analysis}

This section describes the procedures for conducting the local and statistical analysis of the morphology of the Allen-Cahn structure and its evolution.

\subsection{Local Analysis}

Local analysis focuses on the evolution of a particular region of interfaces. Local analysis is carried out by mapping the interfacial properties, such as the mean and Gaussian curvatures and their time derivatives, on the surface of a small portion of the Allen-Cahn 
structure. The microstructure shown in Fig. 1, which is in a cubic domain with side lengths of $6.2\left(S_{v}^{-1}\right)$, is analyzed.

\subsection{Statistical Analysis}

Statistical analysis quantifies the overall morphology the Allen-Cahn structure and its evolution. For this analysis, the entire structure is examined. We apply two statistical characterization techniques: the ISD superimposed with average curvature velocity arrows and the probability contour map of time derivatives of mean curvature as a function of $H$ and of $H$ and $K$.

The interfacial shape distribution is the probability, $P_{I S D}\left(\kappa_{1}, \kappa_{2}, t\right)$, of finding an interface with a given pair $\kappa_{1}$ and $\kappa_{2}$ at time $t$ :

$$
P_{I S D}\left(\kappa_{1}, \kappa_{2}, t\right)=\frac{A_{I S D}\left(\kappa_{1}, \kappa_{2}, t\right)}{A_{T}(t)},
$$

where $A_{I S D}\left(\kappa_{1}, \kappa_{2}, t\right)$ is the total surface area of all interfaces with given principal curvatures and $A_{T}$ is the total surface area of the structure at time $t$. This is numerically evaluated by summing interfacial areas that have curvatures within the range of $\kappa_{1} \pm \Delta \kappa_{1} / 2$ and $\kappa_{2} \pm \Delta \kappa_{2} / 2$, where $\Delta \kappa_{1}$ and $\Delta \kappa_{2}$ are the bin sizes in $\kappa_{1}$ and $\kappa_{2}$, respectively. The bin sizes are chosen such that there is sufficient statistics within the bin while providing sufficient resolution on the curvature space. The same approach is taken for all probability distribution calculations discussed later. Fig. 2a describes the interfacial morphologies in the principal curvature space. Labels '1-4' mark the four regions of the ISD that represent different interfacial morphologies. Regions 1 and 4 represent elliptic interfaces and regions 2 and 3 represent saddle-shaped (hyperbolic) interfaces. The $H=\left(\kappa_{1}+\kappa_{2}\right) / 2=0$ line represents symmetric saddle-shaped interfaces. In addition, curvature coordinates farther away from the origin of the ISD represent interfaces with higher net curvatures, $d=\left(\left(\kappa_{1}^{2}+\kappa_{2}^{2}\right) / 2\right)^{1 / 2}$, which represent the overall bend of a surface. For an interface with spherical geometry, the net curvature reduces to the inverse of the radius of the particle, $1 / R$.

To understand how, on average, the morphologies of the interfaces with the same principal curvatures evolve, we calculate the average velocity in the curvature space, $\vec{v}_{\boldsymbol{\kappa}}$, at each 
curvature coordinate:

$$
\vec{v}_{\kappa}=\left\langle\dot{\kappa}_{1}\right\rangle \hat{\kappa}_{1}+\left\langle\dot{\kappa}_{2}\right\rangle \hat{\kappa}_{2},
$$

where $\left\langle\dot{\kappa}_{1}\right\rangle$ and $\left\langle\dot{\kappa}_{2}\right\rangle$ are the area-averages of the time derivatives of principal curvatures of all interfaces with given $\kappa_{1}$ and $\kappa_{2}$, and $\hat{\kappa}_{1}$ and $\hat{\kappa}_{2}$ are the unit vector on the principal curvature space. The vector, $\vec{v}_{\kappa}$, is represented as arrows superimposed on the ISD (termed "average curvature velocity arrows"). The direction of the curvature velocity arrow is determined by the direction of $\vec{v}_{\kappa}$ and the length of the arrow is representative of $\left|\vec{v}_{\kappa}\right|$.

The second characterization technique is the probability contour map. The evolution of mean curvature is statistically characterized by the probability distribution, $P_{1}(H, \dot{H})$, defined as

$$
P_{1}(H, \dot{H})=\frac{A_{1}(H, \dot{H})}{A_{T}},
$$

where $A_{1}(H, \dot{H})$ is the total surface area of all interfaces with given $H$ and $\dot{H}$. This is evaluated numerically as described earlier. Additionally, to investigate the role of $K$, which together with $H$ determine the interfacial morphology, in the evolution of mean curvature, we calculate the probability distribution, $P_{2}(H, K, \dot{H})$, defined as

$$
P_{2}(H, K, \dot{H})=\frac{A_{2}(H, K, \dot{H})}{A_{T}},
$$

where $A_{2}(H, K, \dot{H})$ is the total surface area of all interfaces with given $H, K$, and $\dot{H}$.

\section{Results}

\subsection{Local Analysis}

The interfaces colored by the mean and the Gaussian curvature values are shown on Fig. 3. The bicontinuous Allen-Cahn structure is composed of spatially varying curvatures with interfacial morphologies that continuously vary from elliptical to cylindrical and to hyperbolic (saddle-shaped) interfaces. The surface plots also show many evidence of topological singularities in the form of pinching. The numbers marked on Fig. 3b highlight regions of interfaces that are about to undergo pinching (labels (1) and (2)) and regions of interfaces 
that are remnant of pinching (labels (3) and (4)). We find that interfaces that are about to pinch off always have large $|H|(>\sim 5$, which can still be tracked with the given diffuse interface) and negative $K$ values (asymmetric saddle-shaped interfaces) and interfaces that are remnant of pinching have large $|H|$ and positive $K$ values (elliptic interfaces). The effect of topological singularities on the evolution of interfacial curvatures of the Allen-Cahn structure will be discussed in Section 4.2.

As a next step in the local analysis, we investigated the local dynamics of interfaces through the calculations of the interfacial velocity and the time derivatives of curvatures, using the Allen-Cahn structure at different simulation times. In the limit where the interfacial thickness is much smaller than the radius of curvature of the interface, the interfacial velocity, $v$, in Allen-Cahn dynamics is propertional to the mean curvature of the interface:

$$
v=-M H
$$

where the proportionality constant, $M$, is given by $M=2 \epsilon^{2} L$, where $\epsilon$ and $L$ are the phase field simulation parameters (see Eq. (1)). To verify this local dependence of the interfacial velocity, we also calculated the interfacial velocity as a function of mean curvature, the right-hand side of Eq. (10). Fig. 4 shows excellent agreement between the two velocity data, one from the time derivative of the smoothed level-set function and the other based on the right-hand side of Eq. (10). This agreement demonstrates the accuracy of the method employed to calculate the interfacial velocity from the microstructural data at two closely separated times. This also demonstrates that, for most of the interfacial area, the nonzero interfacial thickness has no effect on the interfacial velocities.

The evolution of interfacial curvatures, on the other hand, is examined by the calculation of the time derivatives of mean and Gaussian curvatures, $\dot{H}$ and $\dot{K}$, respectively. Fig. 5 shows interfaces colored with $\dot{H}$ and $\dot{K}$ values. Compared to the interfacial velocity (Fig. 4), the values of time derivatives of curvatures exhibit much larger fluctuations throughout the interfaces. More importantly, a direct comparison between the surface plots of $H$ and $\dot{H}$ (Figs. 3a \& 5a) reveals a nonlinear relationship between the two quantities, even though the interfacial velocity is linearly dependent on $H$. 
To gain insight into the evolution of the mean curvature via nonconserved dynamics, we examine the expression for the time derivative of mean curvature, derived from differential geometry:

$$
\dot{H}=-\left(2 H^{2}-K\right) v-\frac{1}{2}\left(v_{, 11}+v_{, 22}\right),
$$

where $v_{, 11}$ and $v_{, 22}$ are the second derivatives of the interfacial velocity with respect to the principal coordinates [53]. This expression clearly shows that there are two contributions to the evolution of mean curvatures. In particular, the first term in Eq. (11) depends solely on the local curvatures and local normal velocity, while the latter depends on the variation of the velocity. Using Eq. (10), Eq. (11) can be re-written as

$$
\dot{H}=M\left(2 H^{2}-K\right) H+\frac{M}{2}\left(H_{, 11}+H_{, 22}\right) .
$$

Therefore, for nonconserved dynamics, the first term only depends on the local interfacial curvatures, $M\left(2 H^{2}-K\right) H$, and the second term depends on the variation of mean curvature along the surface, $\frac{M}{2}\left(H_{, 11}+H_{, 22}\right)$. Note that this term is the surface Laplacian of the mean curvature, and in the absence of the local term, the evolution equation, Eq. (12), reduces to the surface diffusion equation, which typically describes the concentration of a chemical species diffusing on a surface. Hereafter, we refer to the first term in Eq. (12) as the local term and the second term as the nonlocal term.

Since $M$ and $\left(2 H^{2}-K\right)$ are positive, the local term of $\dot{H}$ always has the same sign as the mean curvature. Therefore, the local term, which consists of only $H$ and $K$, do not induce reduction of $|H|$ (the magnitude of $H$ ), which would be required to flatten an interface. On the other hand, the nonlocal term of $\dot{H}$ is has the form of the surface Laplacian of $H$, which tends to smooth it in the manner similar to surface diffusion of chemical species. Hence, the nonlocal term drives $H$ toward values similar to the surface's neighbors. Depending on the values of $H$ in the neighborhood, the resulting change may be in the direction of increasing or decreasing $|H|$. However, since this structure has an average mean curvature of zero, the overall tendency is toward decreasing $|H|$. This prediction of the role of the local and nonlocal term in $\dot{H}$ is validated by Fig. 6 , which shows the interfaces colored by $H$, the local term, and the nonlocal term in Eq. (12). By comparing Figs. 6a and b, one can observe that 
the high (positive) mean curvature region (in red in Fig. 6a) has positive value of the local term (in red in Fig. 6b). On the other hand, the signs of the nonlocal term (Fig. 6c) tend to be opposite of the signs of the mean curvatures, but not always. This is because the term depends on how the curvature varies (and thus depends on the neighboring curvatures). Because of this term, even in the case of nonconserved dynamics, the interfacial curvature evolution cannot be described by the local curvatures, even though the interfacial velocity is the sole function of the local value of $H$ in this dynamics.

\subsection{Statistical Analysis}

The ISD of the Allen-Cahn structure in a scaled principal curvature space is shown in Fig. 7 (both plots show the same ISD, and the arrows superimposed on the ISD are discussed below). It shows that the most of the distribution is in the regions 2 and 3 of the ISD, which indicates that the majority of interfaces are saddle-shaped. The symmetry of the ISD about the $H=0$ line $\left(\kappa_{1}=-\kappa_{2}\right)$ is a consequence of the 50:50 phase fraction of the Allen-Cahn structure, the steady-state structure arising from nonconserved dynamics. Note that a 50:50 phase fraction alone is not sufficient for achieving the symmetry of the ISD about the $H=0$ line; in the case of Allen-Cahn structure, the symmetry between the two phases is ensured by the dynamics and the initial condition that do not bias either of the phases.

Fig. 7a shows the average curvature velocity arrows on the ISD, which represent the velocity (including the magnitude) in curvature space, $\vec{v}_{\boldsymbol{\kappa}}$ in Eq. (7). Fig. $7 \mathrm{~b}$ is presented to show the direction of the curvature velocity by plotting the normalized average curvature velocity arrows, $\vec{v}_{\boldsymbol{\kappa}} /\left|\vec{v}_{\boldsymbol{\kappa}}\right|$, as it is difficult to show the direction in Fig. 7a when the value of $\vec{v}_{\boldsymbol{\kappa}}$ is small. The average curvature velocity arrows demonstrate that the magnitude of $\vec{v}_{\boldsymbol{\kappa}}$ is much greater at curvature coordinates far away from the origin (interfaces with large net curvatures) than it is at curvature coordinates closer to the origin. This implies that highly curved interfaces of the Allen-Cahn structure are rapidly changing their interfacial morphologies during coarsening, as expected.

Fig. $7 \mathrm{~b}$ shows that the normalized average curvature velocity arrows about the peak of the distribution point toward lower net curvature coordinates (i.e. the majority of interfaces 
are flattening), but most of the arrows at coordinates far away from the origin of the ISD point toward higher net curvature coordinates. This tendency indicates that large portions of highly curved interfaces are increasing their net curvatures during coarsening, even though the overall net curvature decreases (or alternatively, the characteristic length scale increases).

This phenomenon is not unlike those observed in coarsening in particulate systems. In a system of spherical particles, the average particle size increases only by disappearance of small particles. These particles, which are smaller than the critical particle size, shrink and their curvatures increases in the process. The coarsening thus proceeds by two concurrent processes: smaller particles increasing their net curvatures, evolving towards topological singularity (disappearance), and larger particles growing at their expense. An analogy can be drawn for the bicontinuous structure. In this case, the reduction of the overall net curvature during coarsening is accomplished by : a) some of the small features increasing in net curvatures and evolving toward topological singularity (in this case, pinching) and b) large features flattening. Unlike the spherical particle systems, however, some of the small features (with high net curvatures) also flatten when they are surrounded by flatter interfaces due to the nonlocal term. This is the case subsequent to pinching, where the remnant is retracting.

All of these processes can be observed in Fig. 7b. The normalized average curvature velocity arrows that point to higher net curvatures in regions 2 and 3 of the ISD on Fig. $7 \mathrm{~b}$ represent the saddle-shaped interfaces increasing their net curvatures as they evolve toward pinching. On the other hand, the nearly spherical interfaces with high curvatures, likely a remnant of pinching, evolve toward the origin (i.e., flattening), as indicated by the arrows pointing toward lower net curvatures in regions 1 and 4 close to the $\kappa_{1}=\kappa_{2}$ line. These results show that topological singularities play a vital role in increasing the length scale of the Allen-Cahn structure during coarsening.

We also investigate the statistical dispersions of $\dot{H}$, as well as its local term and its nonlocal term for a given $H$ value. Fig. 8 shows the probability contour maps of these quantities. Fig. 8a shows the probability distribution of for a given set of $\dot{H}$ and $H$; the red indicates larger area of interfaces of having the set of $\dot{H}$ and $H$, and black indicates little 
or no area. If these two quantities are perfectly correlated, the distribution will collapse to a line. Fig. 8a indicates some correlation (trend), but with a large dispersion. Figs. 8b and c show corresponding maps for the local and nonlocal terms. By comparing the three figures, it is evident that much of the spread of $\dot{H}$ values for a given $H$ value stems from the nonlocal term. The probability contour map of the local term of $\dot{H}$ shows a positive and tight correlation between the local term and the mean curvature (i.e., local term always increases $|H|$ and spread as long as the value of $K$ is not large). Note that the probability in Fig. 8b is strongly peaked due to the tight correlation, and thus the color bar range is significantly different from Figs. 8a and c. In contrast, the probability contour map of the nonlocal term of $\dot{H}$ (Fig. 8c) shows, on average, a negative correlation between the nonlocal term and the mean curvature (i.e., the nonlocal term preferentially decrease $|H|$ ). Hence the three probability contour maps on Fig. 8 reaffirm the conclusion from the local analysis that the local term of $\dot{H}$ increases $|H|$, while the nonlocal term favors decrease in $|H|$, but with a large dispersion.

While the source of the dispersion of the local term is clear, the source of the dispersion in the nonlocal term is not. To examine whether the dispersion originates from its dependence on $K$, we plot the probability contour map for the nonlocal term for fixed $K$ values, $K=$ $-4,-1,0$, and 1 , in Fig. 9. Interfaces with negative $K$ are those that lie in the regions 2 and 3 of the ISD map $\left(\kappa_{1}<0, \kappa_{2}>0\right)$, as shown in Fig. 2b. These saddle-like shapes constitute the majority of interfaces in the structure. The distributions of the nonlocal term of $\dot{H}$ are alike in this class of interfaces, which are similar to that of the entire structure. The interfaces with $K=-4$ have similar morphologies as those with $K=-1$ but differ by the magnitude of $K$. The larger spread in the nonlocal term for interfaces with $K=-4$ indicates that the immediate neighborhood of the interfaces have more diverse interfacial morphologies for saddle-like interfaces with larger $|K|$ than those with smaller $|K|$. The $K=0$ interfaces have cylindrical shapes. It consists of two populations, one with $\kappa_{1}=0$ and the other with $\kappa_{2}=0$, whose distributions for the nonlocal term of $\dot{H}$ are antisymmetric. The combined distribution is still reminiscent of those of the interfaces with negative $K$. On the other hand, the distribution for $K=1$ is significantly different. It consists of two distinct 
distributions, one belonging to the region 1 of ISD $\left(\kappa_{1}>0, \kappa_{2}>0\right)$, and the other region $4\left(\kappa_{1}<0, \kappa_{2}<0\right)$. These interfaces have elliptic shapes, and exhibit a wider spread in the nonlocal term than those with $K=-1$. Nevertheless, it follows the same trend as other populations in that the nonlocal term is negatively proportional to $H$.

In all of these probability plots shown in Fig. 9, the large dispersion in the nonlocal term persists, indicating that the spread in $K$ for given $H$ is not responsible for the dispersion observed in Fig. 8c. Therefore, the origin of the dispersion is truly nonlocal, and it cannot be characterized by the local curvatures or shape of a patch of interface. This nonlocality is a result of the surface diffusion term in the evolution equation for $H$, Eq. (12).

\section{Conclusion}

We investigated the morphological evolution of a bicontinuous structure simulated via nonconserved dynamics to elucidate the complex dynamics of coarsening. A two-phase bicontinuous structure was generated using the phase-field method. The resulting order parameter describing the interfaces of the Allen-Cahn structure was smoothed by the level-set smoothing method (previously developed by the authors) so that the interfacial properties, including time-derivative quantities, could be accurately calculated. These interfacial properties were then used to quantify the morphology and its evolution to understand the dynamics of coarsening of the bicontinuous structure.

In the local analysis, we found many examples of topological singularities in the form of pinching. The interfacial velocity has a simple linear dependence on mean curvature, as expected for nonconserved dynamics. On the other hand, the evolution of mean curvature is much more complex as it depends on both the interfacial curvatures and the curvatures of

the surrounding interfaces. The study of the local and nonlocal terms that contribute to $\dot{H}$ showed that the local term always increases $|H|$, whereas the average value of the nonlocal term decreases $|H|$; the resulting evolution is given by a competition of the two terms. The analysis of the phase field simulation data shows that the nonlocal term is significant and facilitates flattening of the interfaces, in most cases. 
The average curvature velocity arrows superimposed on the ISD demonstrated that, while the majority of interfaces are flattening (decreasing net curvatures), some of highly curved interfaces are increasing their net curvatures. This was attributed to features evolving toward topological singularity (pinching). The probability contour maps of $\dot{H}$, its local term, and its nonlocal term confirmed that the large dispersion of $\dot{H}$ for a given $H$ value stems from the nonlocal term of $\dot{H}$. We also show that the dispersion in the nonlocal term is not due to variation in $K$ for a given $H$. We also identify the tendency of the nonlocal term to smooth the value of $H$ based on the expression for $\dot{H}$, in which the nonlocal term is proportional to the surface Laplacian of $\mathrm{H}$.

This study was enabled by characterization techniques that are based on both static and dynamic interfacial properties. The insights gained from this study will be useful in developing a theory of coarsening of complex microstructures. In particular, we have developed an understanding of the evolution of interfacial morphologies when the normal velocity is known. A different system could be studied by combining the approach presented in this paper with an appropriate description of interfacial velocity.

\section{Acknowledgments}

Authors are grateful for the support from the Department of Energy Office of Basic Energy Science (Grant No. DE-FG02-99ER45782/A012). The computational resources were provided by the Extreme Science and Engineering Discovery Environment (XSEDE), which is supported by National Science Foundation grant number OCI-1053575, under allocation No. TG-DMR110007, as well as the University of Michigan Advanced Research Computing.

\section{References}

[1] A. J. Ardell, R. B. Nicholson, Coarsening of $\gamma^{\prime}$ in Ni-Al Alloys, J. Phys. Chem. Solids 27 (11-1) (1966) 1793-1804, ISSN 0022-3697, doi:10.1016/0022-3697(66)90110-7.

[2] T. Z. Kattamis, J. C. Coughlin, M. C. Flemings, Influence of coarsening on dendrite arm spacing of Aluminum-Copper alloys, Trans. TMS-AIME 239 (10) (1967) 1504-1511, ISSN 0543-5722.

[3] H. A. Calderon, P. W. Voorhees, J. L. Murray, G. Kostorz, Ostwald ripening in concentrated alloys, Acta Metall. Mater. 42 (3) (1994) 991-1000, ISSN 0956-7151, doi:10.1016/0956-7151(94)90293-3. 
[4] T. Pollock, A. Argon, Directional coarsening in nickel-base single crystals with high volume fractions of coherent precipitates, Acta Metall. Mater. 42 (6) (1994) 1859-1874, ISSN 0956-7151, doi:10.1016/09567151(94)90011-6.

[5] C. J. Kuehmann, P. W. Voorhees, Ostwald ripening in ternary alloys, Metall. Mater. Trans. A 27 (4) (1996) 937-943, ISSN 1073-5623, doi:10.1007/BF02649761.

[6] D. Kammer, P. W. Voorhees, The morphological evolution of dendritic microstructures during coarsening, Acta Mater. 54 (6) (2006) 1549-1558, ISSN 1359-6454, doi:10.1016/j.actamat.2005.11.031.

[7] J. Erlebacher, Mechanism of coarsening and bubble formation in high-genus nanoporous metals, Phys. Rev. Lett. 106 (22), ISSN 0031-9007, doi:10.1103/PhysRevLett.106.225504.

[8] T. Philippe, P. W. Voorhees, Ostwald ripening in multicomponent alloys, Acta Mater. 61 (11) (2013) 4237-4244, ISSN 1359-6454, doi:10.1016/j.actamat.2013.03.049.

[9] P. Wiltzius, A. Cumming, Domain growth and wetting in polymer mixtures, Phys. Rev. Lett. 66 (23) (1991) 3000-3003, ISSN 0031-9007, doi:10.1103/PhysRevLett.66.3000.

[10] T. Hashimoto, M. Takenaka, T. Izumitani, Spontaneous pinning of domain growth during spinodal decomposition of off-critical polymer mixtures, J. Chem. Phys. 97 (1) (1992) 679-689, ISSN 0021-9606, doi:10.1063/1.463562.

[11] G. Krausch, C. A. Dai, E. J. Kramer, J. F. Marko, F. S. Bates, Interference of spinodal waves in thin polymer films, Macromolecules (Washington, DC, U. S.) 26 (21) (1993) 5566-5571, ISSN 0024-9297, doi:10.1021/ma00073a006.

[12] S.-W. Song, J. M. Torkelson, Coarsening effects on microstructure formation in isopycnic polymer solutions and membranes produced via thermally induced phase separation, Macromolecules (Washington, DC, U. S.) 27 (22) (1994) 6389-6397, ISSN 0024-9297, doi:10.1021/ma00100a024.

[13] H. Wang, R. J. Composto, Thin film polymer blends undergoing phase separation and wetting: Identification of early, intermediate, and late stages, J. Chem. Phys. 113 (22) (2000) 10386-10397, ISSN 0021-9606, doi:10.1063/1.1322638.

[14] K. B. Glasner, T. P. Witelski, Coarsening dynamics of dewetting films, Phys. Rev. E 67 (1, 2), ISSN 1539-3755, doi:10.1103/PhysRevE.67.016302.

[15] J. Kim, M. K. Gray, H. Y. Zhou, S. T. Nguyen, J. M. Torkelson, Polymer blend compatibilization by gradient copolymer addition during melt processing: Stabilization of dispersed phase to static coarsening, Macromolecules (Washington, DC, U. S.) 38 (4) (2005) 1037-1040, ISSN 0024-9297, doi: $10.1021 / \mathrm{ma0} 47549 \mathrm{t}$

[16] F. M. Ross, J. Tersoff, R. M. Tromp, Coarsening of self-assembled Ge quantum dots on Si(001), Phys. Rev. Lett. 80 (5) (1998) 984-987, ISSN 0031-9007, doi:10.1103/PhysRevLett.80.984.

[17] O. Kienzle, F. Ernst, M. Ruhle, O. G. Schmidt, K. Eberl, Germanium "quantum dots" embedded in 
silicon: Quantitative study of self-alignment and coarsening, Appl. Phys. Lett. 74 (2) (1999) 269-271, ISSN 0003-6951, doi:10.1063/1.123277.

[18] F. Liu, A. H. Li, M. G. Lagally, Self-assembly of two-dimensional islands via strain-mediated coarsening, Phys. Rev. E 87 (12), ISSN 0031-9007, doi:10.1103/PhysRevLett.87.126103.

[19] P. Liu, Y. W. Zhang, C. Lu, Coarsening kinetics of heteroepitaxial islands in nucleationless StranskiKrastanov growth, Phys. Rev. B 68 (3), ISSN 1098-0121, doi:10.1103/PhysRevB.67.035402.

[20] Y. Tu, J. Tersoff, Coarsening, mixing, and motion: The complex evolution of epitaxial islands, Phys. Rev. E 98 (9), ISSN 0031-9007, doi:10.1103/PhysRevLett.98.096103.

[21] C. Heyn, A. Stemmann, A. Schramm, H. Welsch, W. Hansen, A. Nemcsics, Regimes of GaAs quantum dot self-assembly by droplet epitaxy, Phys. Rev. B 76 (7), ISSN 1098-0121, doi: 10.1103/PhysRevB.76.075317.

[22] R. W. Balluffi, S. Allen, W. C. Carter, Kinetics of Materials, John Wiley \& Sons, 2005.

[23] K. P. Young, D. H. Kerkwood, The dendrite arm spacings of aluminum-copper alloys solidified under steady-state conditions, Metall. Trans. A 6 (1) (1975) 197-205, ISSN 0026-086X, doi: 10.1007/BF02673688.

[24] D. G. McCartney, J. D. Hunt, Measurements of cell and primary dendrite arm spacings in directionally solidified aluminium alloys, Acta Metall. 29 (11) (1981) 1851-1863, ISSN 0001-6160, doi:10.1016/00016160(81)90111-5.

[25] J. Alkemper, R. Mendoza, P. W. Voorhees, Morphological evolution of dendritic microstructures, Adv. Eng. Mater. 4 (9) (2002) 694-697, ISSN 1438-1656, doi:10.1002/1527-2648(20020916)4:9;694::AIDADEM694¿3.0.CO;2-M.

[26] R. Mendoza, I. Savin, K. Thornton, P. W. Voorhees, Topological complexity and the dynamics of coarsening, Nat. Mater. 3 (6) (2004) 385-388, ISSN 1476-1122, doi:10.1038/nmat1138.

[27] J. L. Fife, P. W. Voorhees, The morphological evolution of equiaxed dendritic microstructures during coarsening, Acta Mater. 57 (8) (2009) 2418-2428, ISSN 1359-6454, doi:10.1016/j.actamat.2009.01.036.

[28] W. R. Osorio, P. R. Goulart, A. Garcia, G. A. Santos, C. M. Neto, Effect of dendritic arm spacing on mechanical properties and corrosion resistance of Al 9 Wt Pct Si and Zn 27 Wt Pct Al alloys, Metall. Mater. Trans. A 37A (8) (2006) 2525-2538.

[29] P. R. Goulart, W. R. Osório, J. E. Spinelli, A. Garcia, Dendritic microstructure affecting mechanical properties and corrosion resistance of an Al-9 wt\% Si alloy, Mater. Manuf. Processes 22 (3) (2007) $328-332$.

[30] P. W. Voorhees, The theory of Ostwald ripening, J. Stat. Phys. 38 (1-2) (1985) 231-252, ISSN 00224715, doi:10.1007/BF01017860.

[31] I. M. Liftshitz, V. V. Slyozov, The kinetics of precipitation from supersaturated solid solutions, J. Phys. 
Chem. Solids 19 (1-2) (1961) 35-50.

[32] C. Wagner, Theorie der Alterung von Niederschlägen durch Umlösen (Ostwald Reifung), Z. Elektrochem. 65 (7/8) (1961) 581-591.

[33] H. Jinnai, Y. Nishikawa, T. Koga, T. Hashimoto, Direct observation of three-dimensional bicontinuous structure developed via spinodal decomposition, Macromolecules (Washington, DC, U. S.) 28 (13) (1995) 4782-4784, ISSN 0024-9297, doi:10.1021/ma00117a071.

[34] H. Jinnai, Y. Nishikawa, H. Morimoto, T. Koga, T. Hashimoto, Geometrical properties and interface dynamics: Time evolution of spinodal interface in a binary polymer mixture at the critical composition, Langmuir 16 (9) (2000) 4380-4393, ISSN 0743-7463, doi:10.1021/la991024q.

[35] D. Seol, S. Hu, Y. Li, J. Shen, K. Oh, L. Q. Chen, Three-dimensional phase-field modeling of spinodal decomposition in constrained films, Met. Mater. Int. 9 (1) (2003) 61-66, ISSN 1225-9438, doi: 10.1007/BF03027232.

[36] C.-Y. Kuo, S.-L. Su, H.-A. Tsai, Y.-S. Su, D.-M. Wang, J.-Y. Lai, Formation and evolution of a bicontinuous structure of PMMA membrane during wet immersion process, J. Membr. Sci. 315 (1-2) (2008) 187 - 194, ISSN 0376-7388, doi:http://dx.doi.org/10.1016/j.memsci.2008.02.034.

[37] H. Jinnai, T. Hashimoto, D. Lee, S.-H. Chen, Morphological characterization of bicontinuous phaseseparated polymer blends and one-phase microemulsions, Macromolecules (Washington, DC, U. S.) 30 (1) (1997) 130-136, ISSN 0024-9297, doi:10.1021/ma960486x.

[38] S.-H. Chen, S.-M. Choi, The Gaussian curvature of the oil-water interface in an isometric bicontinuous microemulsion, Phys. A (Amsterdam, Neth.) 236 (1-2) (1997) 38-51, ISSN 0378-4371, doi: http://dx.doi.org/10.1016/S0378-4371(96)00403-7.

[39] Y. Kwon, K. Thornton, P. W. Voorhees, Coarsening of bicontinuous structures via nonconserved and conserved dynamics, Phys. Rev. E 75 (2, 1), ISSN 1539-3755, doi:10.1103/PhysRevE.75.021120.

[40] Y. Kwon, K. Thornton, P. W. Voorhees, Morphology and topology in coarsening of domains via non-conserved and conserved dynamics, Philos. Mag. 90 (1-4) (2010) 317-335, ISSN 1478-6435, doi: $10.1080 / 14786430903260701$.

[41] Y. C. Li, R. P. Shi, C. P. Wang, X. J. Liu, Y. Wang, Phase-field simulation of thermally induced spinodal decomposition in polymer blends, Modell. Simul. Mater. Sci. Eng. 20 (7), ISSN 0965-0393, doi:10.1088/0965-0393/20/7/075002.

[42] M. Fiałkowski, R. Hołyst, Morphological changes during the order-disorder transition in the two- and three-dimensional systems of scalar nonconserved order parameters, Phys. Rev. E 66 (2002) 046121, doi:10.1103/PhysRevE.66.046121.

[43] O. Hellwig, A. Berger, E. E. Fullerton, Domain walls in antiferromagnetically coupled multilayer films, Phys. Rev. Lett. 91 (19) (2003) 197203, doi:10.1103/PhysRevLett.91.197203. 
[44] O. Gutfleisch, K.-H. Müller, K. Khlopkov, M. Wolf, A. Yan, R. Schafer, T. Gemming, L. Schultz, Evolution of magnetic domain structures and coercivity in high-performance SmCo 2:17-type permanent magnets, Acta Mater. 54 (4) (2006) 997-1008, ISSN 1359-6454, doi:10.1016/j.actamat.2005.10.026.

[45] J. Erlebacher, I. McCue, Geometric characterization of nanoporous metals, Acta Mater. 60 (17) (2012) 6164-6174, ISSN 1359-6454, doi:10.1016/j.actamat.2012.07.059.

[46] T. Yu, H. Naganuma, D. W. Shi, Y. Ando, X. F. Han, Magnetic Properties and Magnetic Domain Structures Evolution Modulated by $\mathrm{CoFeB}$ Layer in $[\mathrm{Pd} / \mathrm{Co}] / \mathrm{CoFeB} / \mathrm{MgO} / \mathrm{CoFeB} /[\mathrm{Co} / \mathrm{Pd}]$ Perpendicular MTJ Films, IEEE Trans. Magn. 48 (11) (2012) 2812-2815, ISSN 0018-9464, doi: 10.1109/TMAG.2012.2198795.

[47] J. Sethian, Level Set Methods and Fast Marching Methods: Evolving Interfaces in Computational Geometry, Fluid Mechanics, Computer Vision, and Materials Science, Cambridge University Press, Cambridge, UK, second edn., 1999.

[48] S. Osher, J. A. Sethian, Fronts propagating with curvature-dependent speed: algorithms based on Hamilton-Jacobi formulations, J. Comput. Phys. 79 (1) (1988) 12-49, ISSN 0021-9991, doi: 10.1016/0021-9991(88)90002-2.

[49] S. M. Allen, J. W. Cahn, Microscopic theory for antiphase boundary motion and its application to antiphase domain coarsening, Acta Metall. 27 (6) (1979) 1085-1095, ISSN 0001-6160, doi:10.1016/00016160(79)90196-2.

[50] J. W. Cahn, J. E. Hilliard, Free energy of a nonuniform system. I. interfacial free energy, J. Chem. Phys. 28 (2) (1958) 258-267, ISSN 0021-9606, doi:10.1063/1.1744102.

[51] J. W. Cahn, On spinodal decomposition, Acta Metall. 9 (9) (1961) 795-801, ISSN 0001-6160, doi: 10.1016/0001-6160(61)90182-1.

[52] C.-L. Park, P. W. Voorhees, K. Thornton, Application of the level-set method to the analysis of an evolving microstructure, Comput. Mater. Sci. 85 (2013) 46-58, doi:10.1016/j.commatsci.2013.12.022.

[53] D. A. Drew, Evolution of geometric statistics, SIAM J. Appl. Math. 50 (3) (1990) 649-666, ISSN 0036-1399, doi:10.1137/0150038.

[54] R. Mendoza, Morphological and Topological Characterization of Coarsened Dendritic Microstructures, Ph.D. thesis, Northwestern University, 2004. 


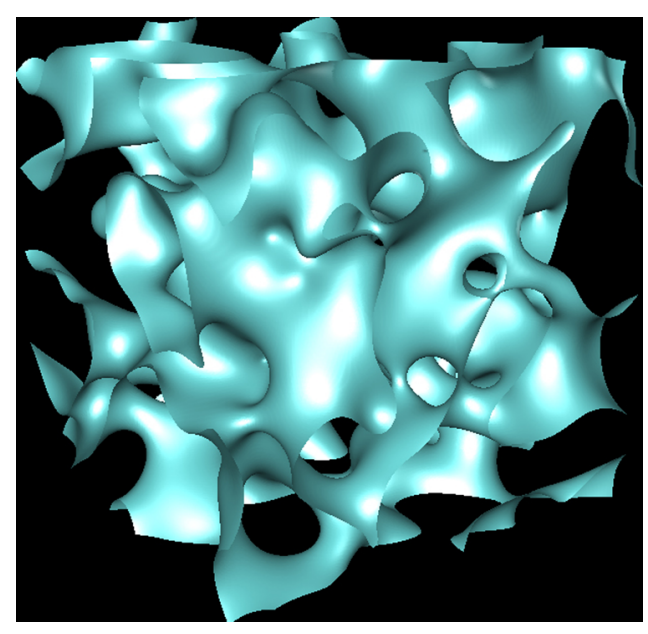

Figure 1: Morphology of the bicontinuous Allen-Cahn structure at the simulation time of $t_{0}=600$. The microstructure shown is in a cubic domain with side lengths of $6.2\left(S_{v}^{-1}\right)$, where $S_{v}$ is the total surface area per unit volume and its inverse is the characteristic length scale. 


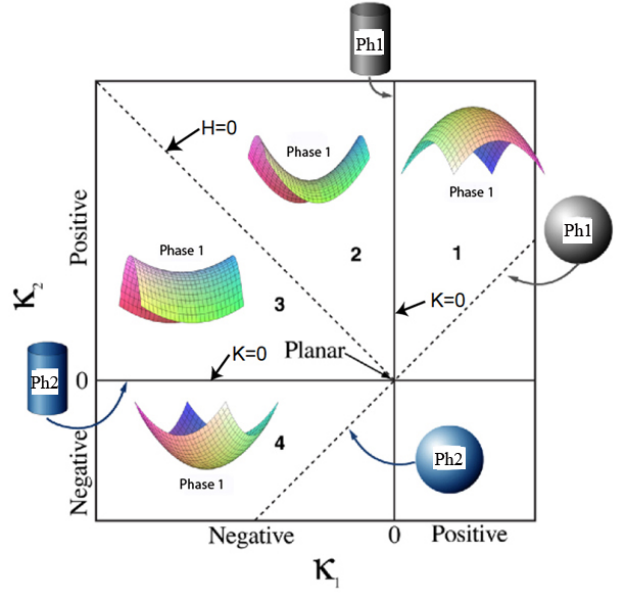

(a)

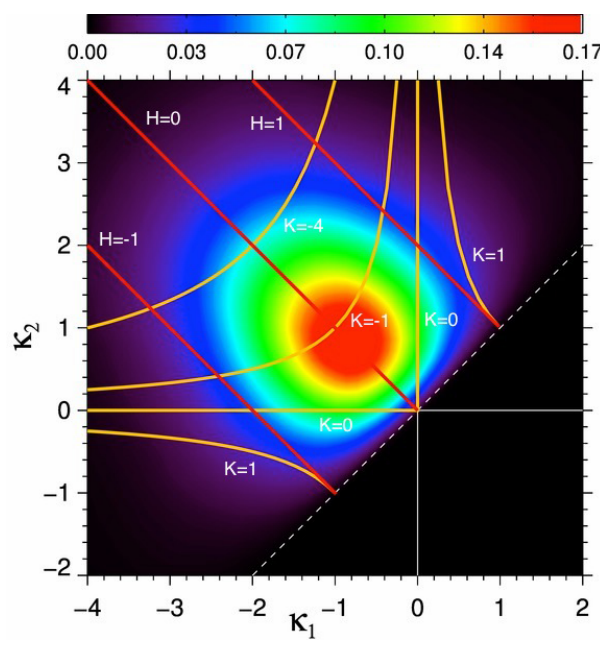

(b)

Figure 2: (a) Graphical representation of interfacial shapes in the principal curvature space where the interfacial shape distribution is defined. Labels 'Ph1' and 'Ph2' represent the two phases in the system. Modified from Ref. [54]. (b) ISD of the Allen-Cahn structure with isolines of constant $H$ and $K$.

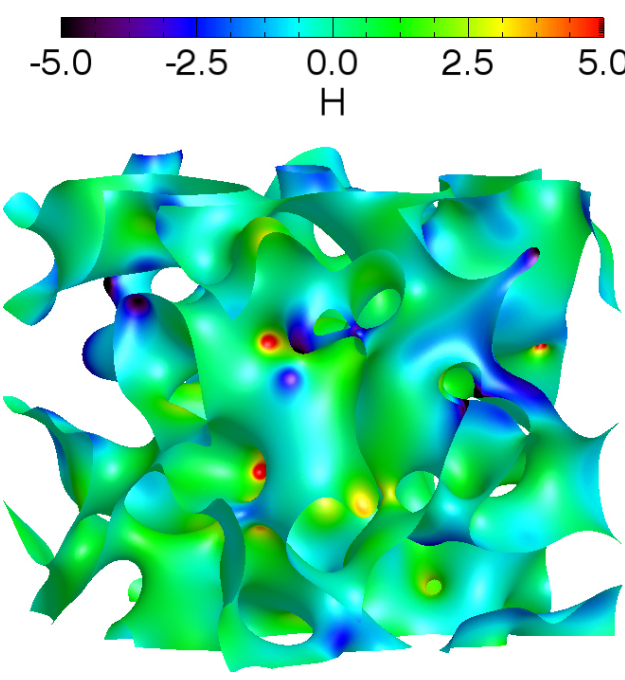

(a)
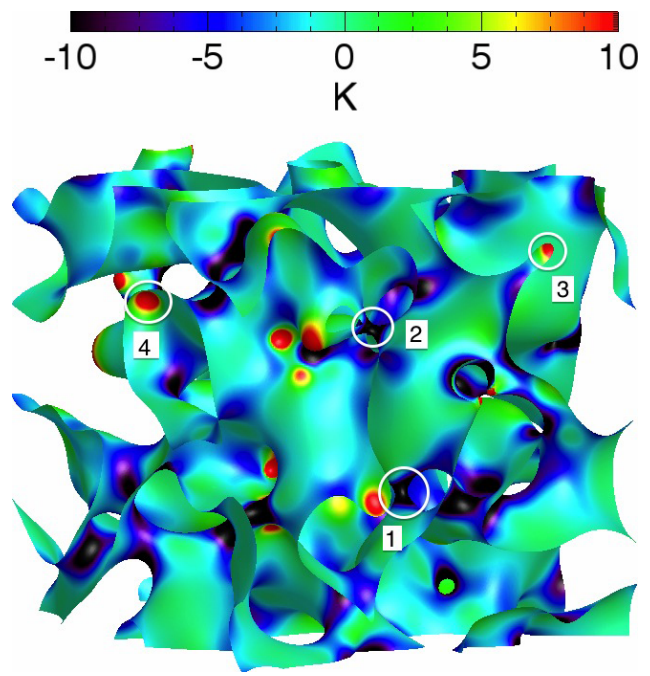

(b)

Figure 3: Interfaces colored by (a) the mean curvature, $H$, and (b) the Gaussian curvature, $K$. Labels (1) and (2) are examples of interfaces that are about to undergo topological singularity (pinching), which always have negative $K$ values (saddle-shaped interfaces). On the other hand, labels (3) and (4) are examples of interfaces that are remnant of pinching, which always have positive $K$ values (elliptic interfaces). 


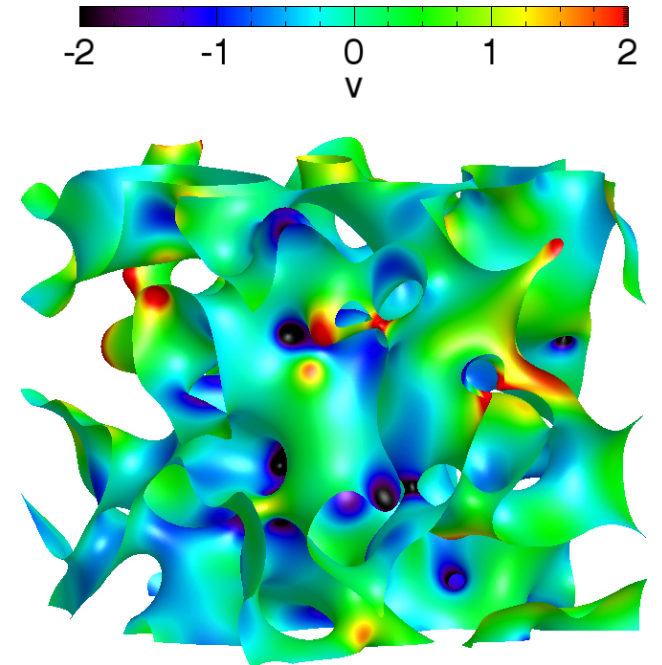

(a)
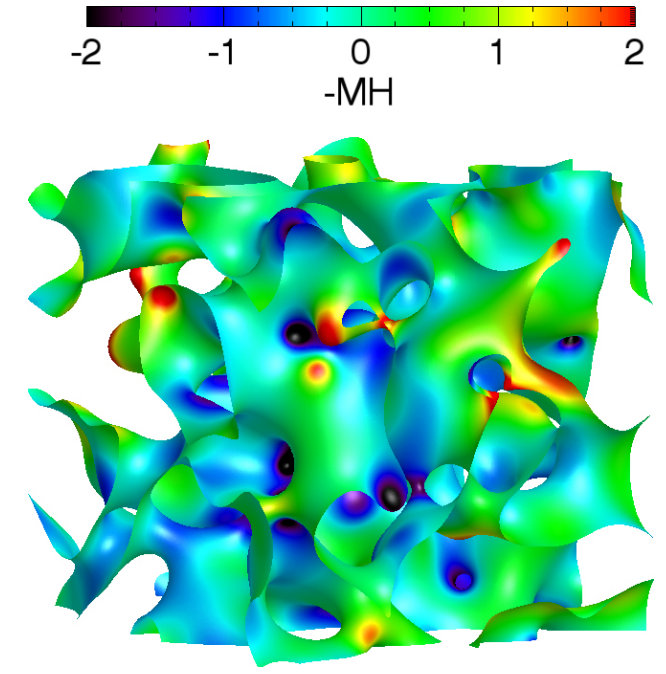

(b)

Figure 4: Interfaces colored by the interfacial velocity calculated from (a) the time derivative of the smoothed level-set function and (b) the mean curvature. The excellent agreement between the two sets of data validates the accuracy of the method employed to calculate the interfacial velocity from the time derivative of the smoothed level-set function.
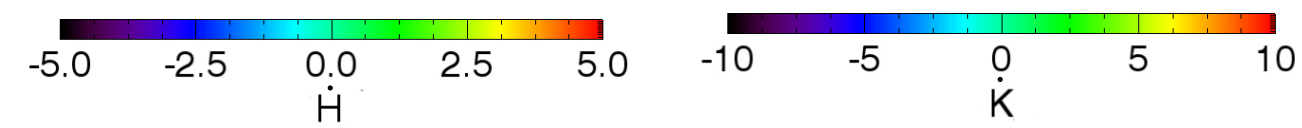

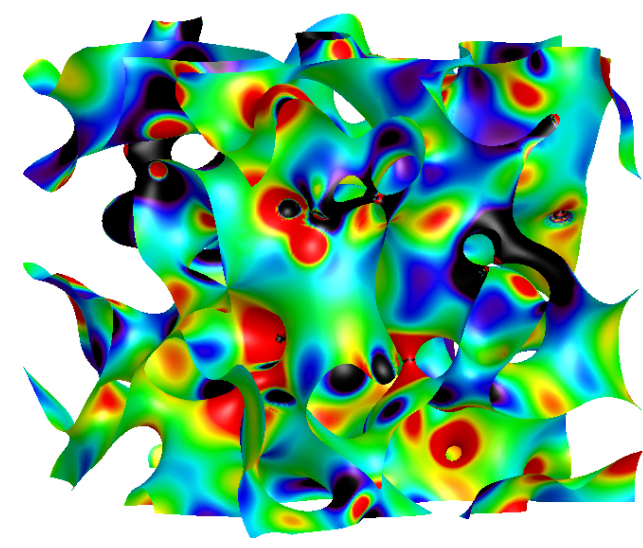

(a)

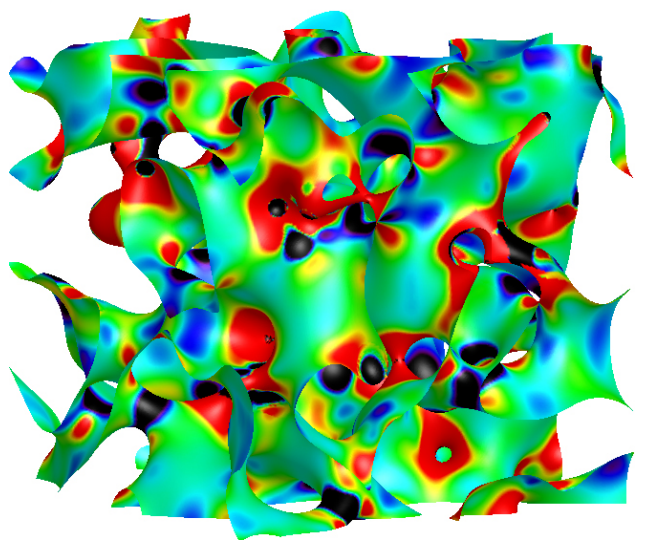

(b)

Figure 5: Interfaces colored by the time derivative of (a) the mean curvature, $\dot{H}$, and (b) the Gaussian curvature, $\dot{K}$. 


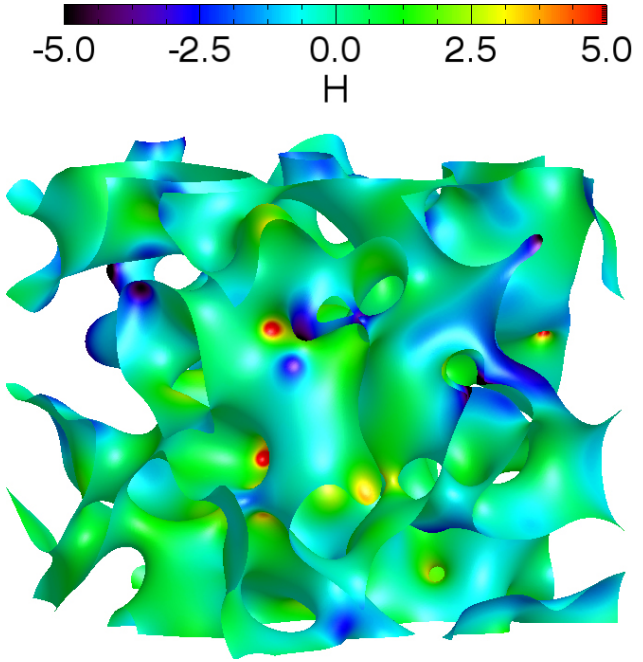

(a)
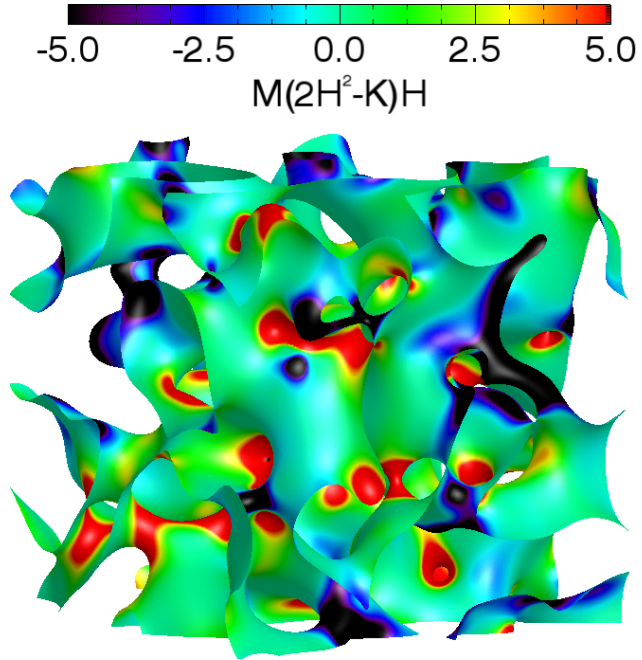

(b)

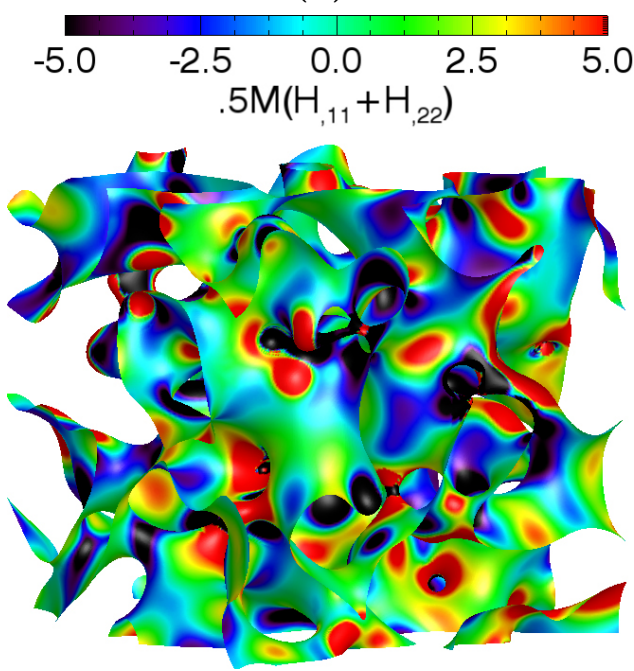

(c)

Figure 6: Interfaces colored by (a) $H$ (b) the local term of $\dot{H}$, and (c) the nonlocal term of $\dot{H}$. The local term always increases the magnitude of $H$ and the nonlocal term favors reduction in the magnitude of $H$. 


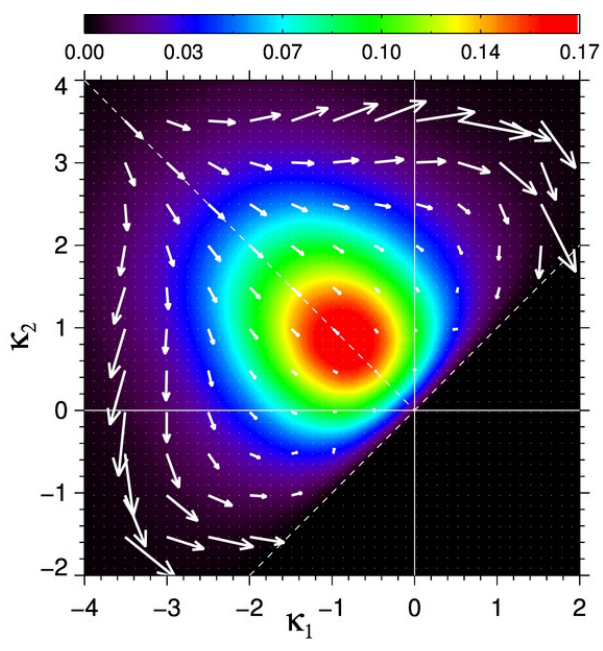

(a)

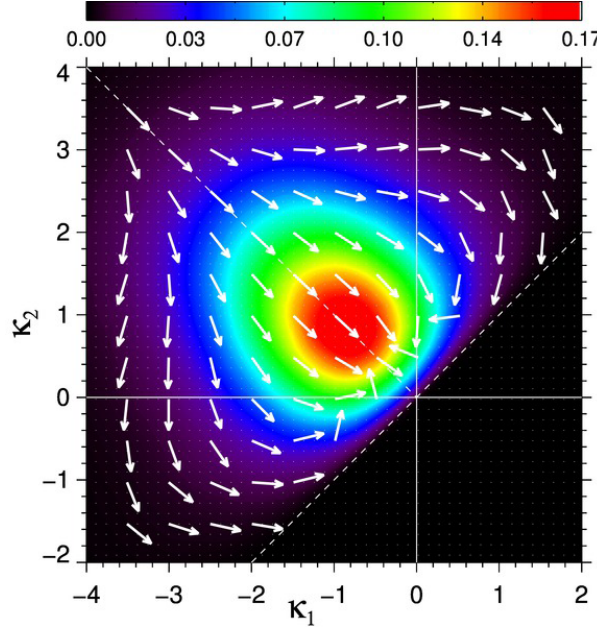

(b)

Figure 7: ISD of the Allen-Cahn structure superimposed with (a) the average curvature velocity arrows, which represent the average velocity in curvature space, $\vec{v}_{\boldsymbol{\kappa}}$, and (b) the normalized average curvature velocity arrows, which represent the normalized average velocity in curvature space, $\vec{v}_{\kappa} /\left|\vec{v}_{\kappa}\right|$. 


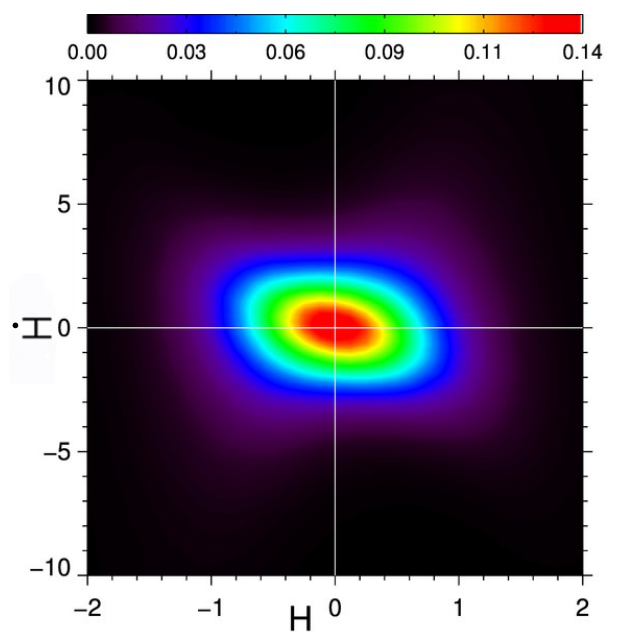

(a)

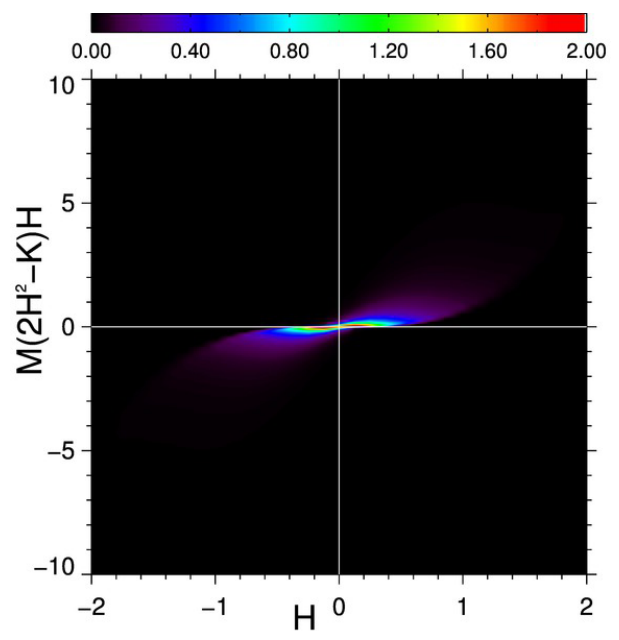

(b)

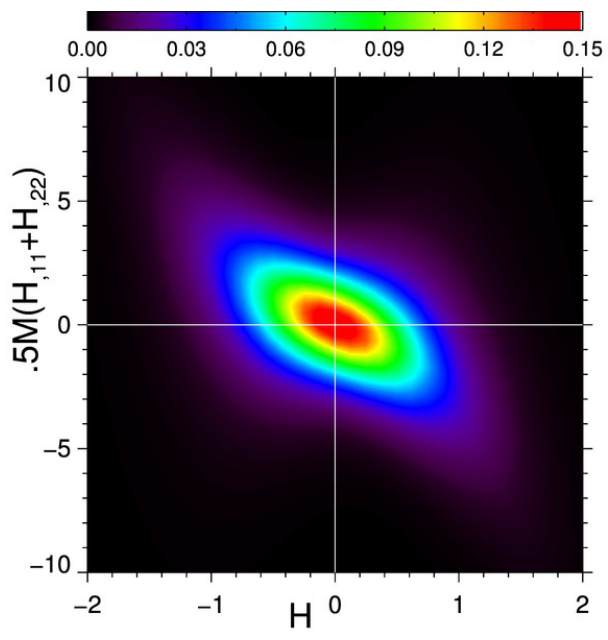

(c)

Figure 8: Probability contour maps of (a) $\dot{H}$ (b) the local term of $\dot{H}$ and (c) the nonlocal term of $\dot{H}$ as functions of $H$. These probability contour maps are consistent with interfacial properties calculations observed locally on Fig. 6. These plots also confirm that much of the dispersion of $\dot{H}$ for a given $H$ value stems from the nonlocal term of $\dot{H}$. 


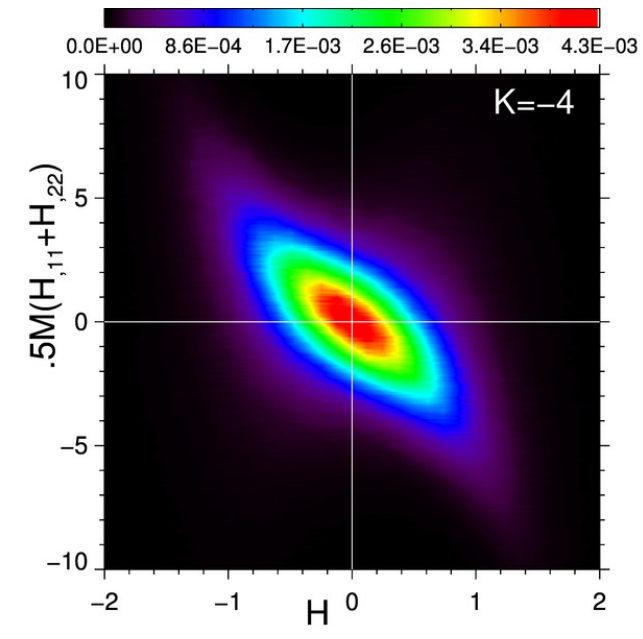

(a)

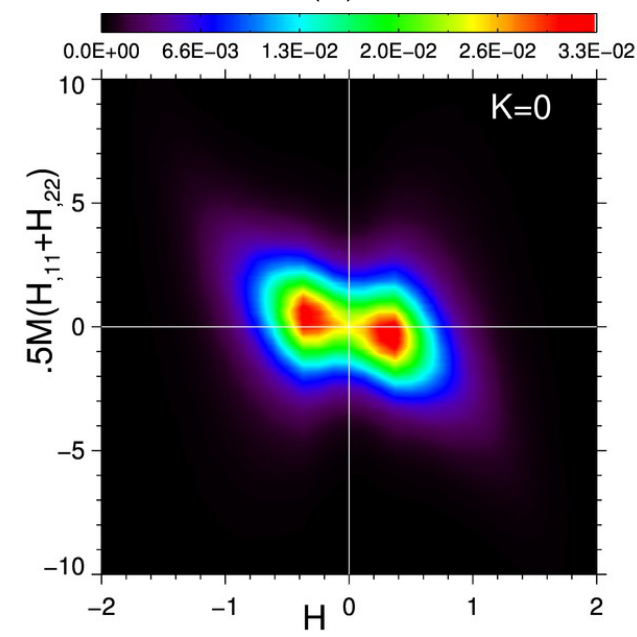

(c)

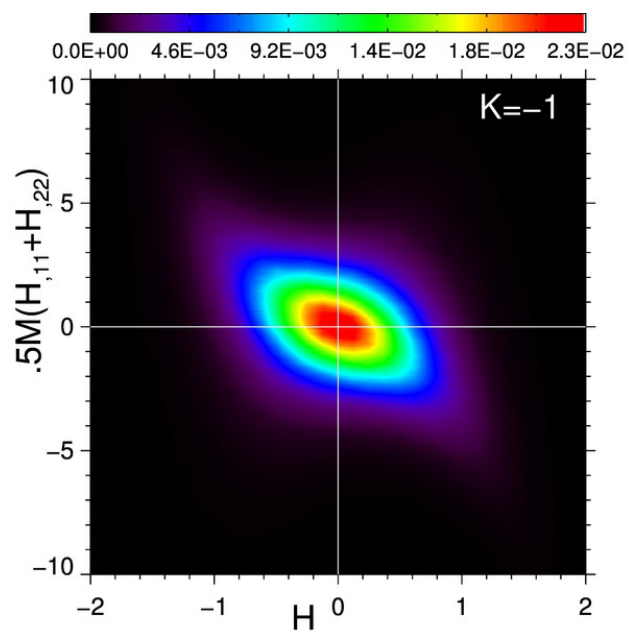

(b)

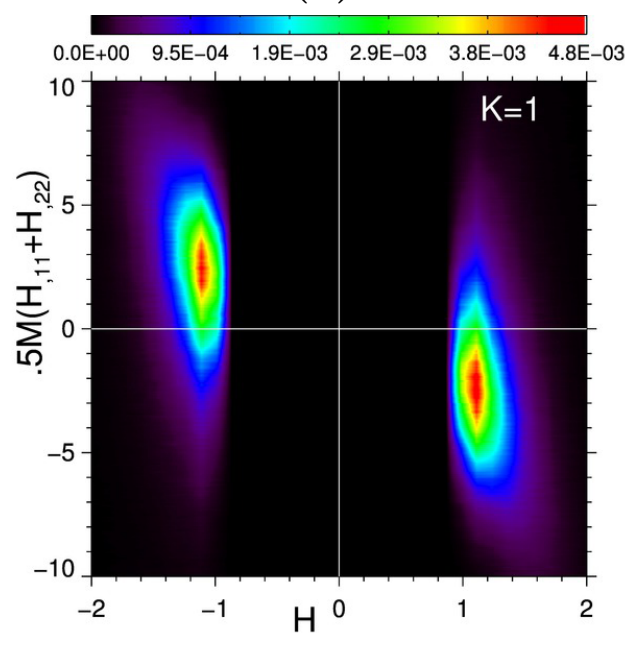

(d)

Figure 9: Probability contour maps of the nonlocal term of $\dot{H}$ as a function $H$ at $K$ values of (a) $K=-4$, (b) $K=-1$, (c) $K=0$, and (d) $K=1$. The large dispersion persists even when the $K$ value is fixed. 